\title{
Maximizing Transfer Efficiency with an Adaptive Wireless Power Transfer System for Variable Load Applications
}

\author{
Jung-Hoon Cho ${ }^{1}$, Byoung-Hee Lee ${ }^{2, *}$ (D) and Young-Joon Kim ${ }^{1, *(\mathbb{D})}$ \\ 1 Department of Electronic Engineering, Gachon University, Seongnam 13120, Korea; jhoonicho@gmail.com \\ 2 Department of Electronic Engineering, Hanbat National University, Daejeon 34158, Korea \\ * Correspondence: bhlee@hanbat.ac.kr (B.-H.L.); youngkim@gachon.ac.kr (Y.-J.K.)
}

Citation: Cho, J.-H.; Lee, B.-H.; Kim, Y.-J. Maximizing Transfer Efficiency with an Adaptive Wireless Power Transfer System for Variable Load Applications. Energies 2021, 14, 1417. https://doi.org/10.3390/en14051417

Academic Editor: William Holderbaum

Received: 20 January 2021

Accepted: 1 March 2021

Published: 4 March 2021

Publisher's Note: MDPI stays neutral with regard to jurisdictional claims in published maps and institutional affiliations.

Copyright: (c) 2021 by the authors. Licensee MDPI, Basel, Switzerland. This article is an open access article distributed under the terms and conditions of the Creative Commons Attribution (CC BY) license (https:// creativecommons.org/licenses/by/ $4.0 /)$.

\begin{abstract}
Electronic devices usually operate in a variable loading condition and the power transfer efficiency of the accompanying wireless power transfer (WPT) method should be optimizable to a variable load. In this paper, a reconfigurable WPT technique is introduced to maximize power transfer efficiency in a weakly coupled, variable load wireless power transfer application. A seriesseries two-coil wireless power network with resonators at a frequency of $150 \mathrm{kHz}$ is presented and, under a variable loading condition, a shunt capacitor element is added to compensate for a maximum efficiency state. The series capacitance element of the secondary resonator is tuned to form a resonance at $150 \mathrm{kHz}$ for maximum power transfer. All the capacitive elements for the secondary resonators are equipped with reconfigurability. Regardless of the load resistance, this proposed approach is able to achieve maximum efficiency with constant power delivery and the power present at the load is only dependent on the input voltage at a fixed operating frequency. A comprehensive circuit model, calculation and experiment is presented to show that optimized power transfer efficiency can be met. A 50 W WPT demonstration is established to verify the effectiveness of this proposed approach.
\end{abstract}

Keywords: magnetic resonant couple; optimization; reconfigurable wireless power transfer; variable load; wireless power transfer

\section{Introduction}

Wireless power transfer (WPT) technique is an enabling technology for many consumer electronics and industrial applications [1]. WPT has been an emerging topic since the 1990s and the progress of WPT technology has led commercialization of wireless chargers for toothbrushes, cellphones and medical implants. Further research activities are pursued to expand WPT technology to power applications, such as continuous power delivery to electronics with dynamic power consumption, while preserving an acceptable power transfer efficiency $[2,3]$.

Energy transfer efficiency is one of the important characteristics in WPT. When the coils are far away from each other or loosely coupled, an optimum condition must be established for maximum power transfer efficiency. This particular condition includes size of inductance, coupling coefficient, resonate frequency and load resistance. Among these conditions, load resistance is often considered variable since most of today's electronic devices have a dynamic loading condition (e.g., brightness change in TV, constant current, constant voltage battery charger, etc.) which indicates that load resistance should be rearranged to the optimum value to achieve maximum energy efficiency. Also, the resistive load under consideration should include electronic appliances with equivalent resistance value higher than $100 \Omega$.

Typically, there are two different approaches for efficiency optimization in a loadvarying application: 
(1) impedance transformation;

(2) direct current (DC)-DC power converters.

The "maximum power transfer" approach has a limited output power, usually less than $10 \mathrm{~W}$, due to the capacity of a power amplifier and the conjugate matching method loses half of its system power efficiency at the source impedance [4-9]. In order to maximize system energy efficiency while delivering high power, a "maximum energy efficiency" approach is pursed in this work. The impedance transformation network typically includes tuning the inductance of the secondary coil $[7,10,11]$, introducing high order compensation network [12-14] or employing intermediate resonator [10,15-17] to transform the load impedance seen by the source for optimization. Employing an impedance transformation network approach in the previous work is effective for fixed load applications but inductance adjustment is usually difficult, and the overall system size becomes bulky for physical implementation of reconfigurable magnetic coupled coil inductance. Moreover, introducing higher order compensation circuit or intermediate resonator demands an increased overall system size and loss. Some researchers utilize a DC-DC converter to regulate the effective load $[18,19]$. This approach can effectively handle dynamic loading condition, but additional converter loss and complicated control circuit is inevitable.

In this paper, a simple but effective impedance transformation technique that requires only one additional capacitor element with reconfigurability is presented. Maximum efficiency over a wide range of loading condition can be achieved without using a DC-DC converter or any complicated compensation network. Unlike the other impedance transformation networks, the proposed approach does not require inductance reconfigurability, which makes the system feasible for practical application. This work only uses reconfigurable capacitors, and the inductance of the magnetic coupled coil remains unchanged, which significantly simplifies the system design and implementation. The additional capacitor is placed parallel to the load and when the resistance of the load increases, the impedance of the load can be transformed to a lower value, placing the system at optimized efficiency. Although, a maximum efficiency is achieved, the resonant frequency of the secondary resonator is altered due to the additional capacitance. Hence, the frequency mismatch is resolved by introducing tunable series capacitance of the secondary side. This proposed approach establishes an optimum efficiency over a wide range of load conditions without introducing complicated circuit and the transferred power is independent of the load resistance.

This paper is organized as follows. The conventional compensation techniques for a variable-load WPT system are discussed. Then the proposed approach with reconfigurable capacitor network is presented. Mathematical derivation and calculation are used to show that maximum efficiency and constant power transfer is achieved at the same time. A prototype of the proposed system is fabricated and measured for verification.

\section{Conventional Impedance Transformation Technique}

The conventional series-series magnetic coupled wireless power transfer system is shown in Figure 1. The parasitic resistance of the resonators is denoted by $R_{p}$. Assuming the resonant frequency is equal $\left(f_{0}=1 / 2 \pi \sqrt{L_{1} C_{1}}=1 / 2 \pi \sqrt{L_{2} C_{2}}\right)$, we can express the secondary circuitry to a reflected impedance, $Z_{r e f}$, and the power transfer efficiency as Equations (1) and (2).

$$
\begin{gathered}
Z_{r e f}=\frac{\omega_{0}^{2} M^{2}}{R_{p 2}+R_{L}} \\
\eta=\frac{Z_{r e f}}{R_{p 1}+Z_{r e f}} \cdot \frac{R_{L}}{R_{p 2}+R_{L}}
\end{gathered}
$$




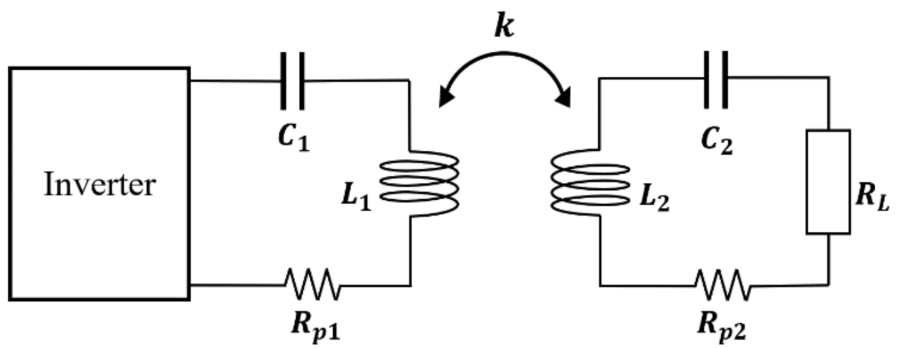

(a)

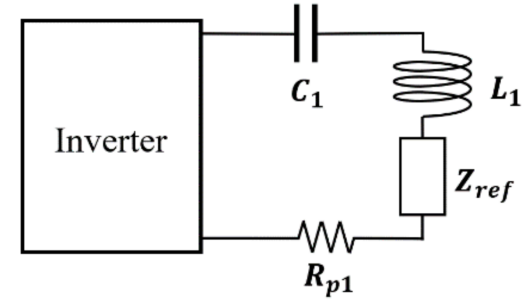

(b)

Figure 1. (a) The circuit model of a conventional wireless power transfer (WPT) system. (b) Equivalent circuit model of the secondary resonator represented by a reflected impedance, $Z_{\text {ref }}$.

It is known that there exists a specific load resistance that can maximize the power transfer efficiency [20]. Assuming the resonate frequency of the resonators are equally $150 \mathrm{kHz}$ and the two coils are identical with an inductance of $100 \mu \mathrm{H}$, quality factor of 460 , the optimum load can be expressed by the following equations. The coupling coefficient between the coils is assumed as 0.02 .

$$
\begin{gathered}
\frac{\partial \eta}{\partial R_{L}}=0 \\
R_{L_{-} o p t}=R_{p 2} \sqrt{1+\frac{\omega_{0}^{2} M^{2}}{R_{p 1} R_{p 2}}}
\end{gathered}
$$

The power transfer efficiency with respect to the load, $R_{L}$, is illustrated as Figure 2. When the two coils are strongly coupled, the transfer efficiency is close to the maximum at a very wide range of loading condition. As the coupling strength becomes weaker, the transfer efficiency drops dramatically, and the performance is highly dependent on the load resistance. It can be seen that, given the parameters in this design, the optimum efficiency occurs at a load resistance of $1.9 \Omega$. In order to establish a maximum efficiency at a higher resistive load, the inductance of the secondary resonator has to increase. Using the following equations, (1) and (2) can be solved as (6) and (7).

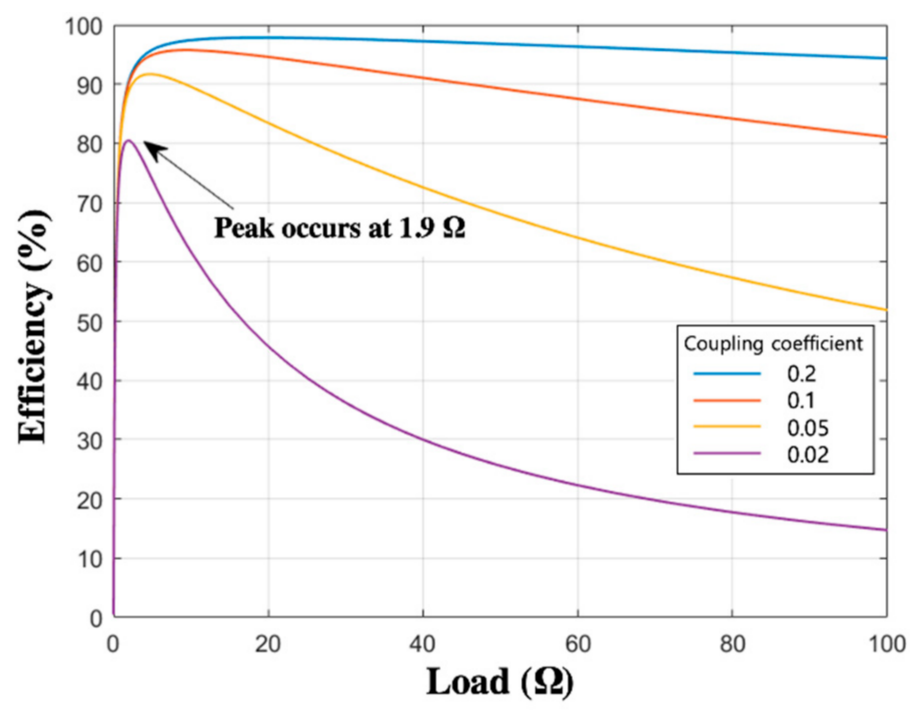

Figure 2. The power transfer efficiency of a conventional WPT system with a variable loading condition at different coupling coefficient values. 


$$
\begin{gathered}
M=k \sqrt{L_{1} L_{2}}, \quad R_{p}=\frac{\omega_{0} L}{Q} \\
Z_{r e f}=\frac{\omega^{2} k^{2} L_{1} L_{2}}{\frac{\omega L_{2}}{Q_{2}}+R_{L}} \\
\eta=\frac{\frac{\omega_{0}^{2} k^{2} L_{2}}{\omega_{0} L_{2}} Q_{2}+R_{L}}{\frac{\omega_{0}}{Q_{1}}+\frac{\omega_{0}^{2} k^{2} L_{2}}{\frac{\omega_{0} L_{2}}{Q_{2}}+R_{L}}} \cdot \frac{R_{L}}{\frac{\omega_{0} L_{2}}{Q_{2}}+R_{L}}
\end{gathered}
$$

By rearranging Equation (7), following equation can be obtained.

$$
\eta=\frac{Q_{1} Q_{2} \omega_{0}^{2} k^{2} R_{L}}{\frac{\omega_{0}^{3} L_{2}}{Q_{2}}+\frac{Q_{1} Q_{2} \omega_{0}^{3} k^{2} L_{2}}{Q_{2}}+\omega_{0}^{2} R_{L}+\omega_{0}^{2} R_{L}+Q_{1} Q_{2} \omega_{0}^{2} k^{2} R_{L}+\frac{Q_{2} \omega_{0} R_{L}^{2}}{L_{2}}}
$$

The optimum inductance required for a maximum efficiency occurs when

$$
\begin{gathered}
\frac{\partial \eta}{\partial L_{2}}=\frac{-Q_{1} Q_{2} \omega_{0}^{2} k^{2} R_{L}\left(\frac{\omega_{0}^{3}}{Q_{2}}+\frac{Q_{1} Q_{2} \omega_{0}^{3} k^{2}}{Q_{2}}-\frac{Q_{2} \omega_{0} R_{L}^{2}}{L_{2}^{2}}\right)}{\left\{\frac{\omega_{0}^{3} L_{2}}{Q_{2}}+\frac{Q_{1} Q_{2} \omega_{0}^{3} k^{2} L_{2}}{Q_{2}}+\omega_{0}^{2} R_{L}+\omega_{0}^{2} R_{L}+Q_{1} Q_{2} \omega_{0}^{2} k^{2} R_{L}+\frac{Q_{2} \omega_{0} R_{L}^{2}}{L_{2}}\right\}^{2}}=0 \\
\frac{\omega_{0}^{3}+Q_{1} Q_{2} \omega_{0}^{3} k^{2}}{Q_{2}}=\frac{Q_{2} \omega_{0} R_{L}^{2}}{L_{2}^{2}} .
\end{gathered}
$$

The optimum inductance can be thus obtained as Equation (11).

$$
L_{2 \_o p t}=\frac{Q_{2} R_{L}}{\omega_{0} \sqrt{1+k^{2} Q_{1} Q_{2}}}
$$

Solving (9) for $L_{2}$, we can obtain Figure 3.

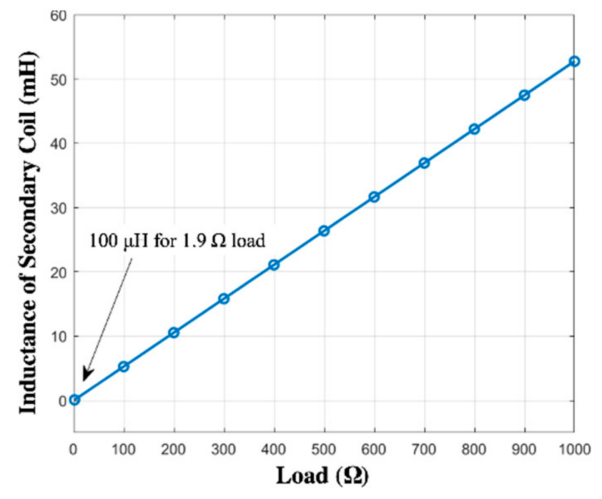

(a)

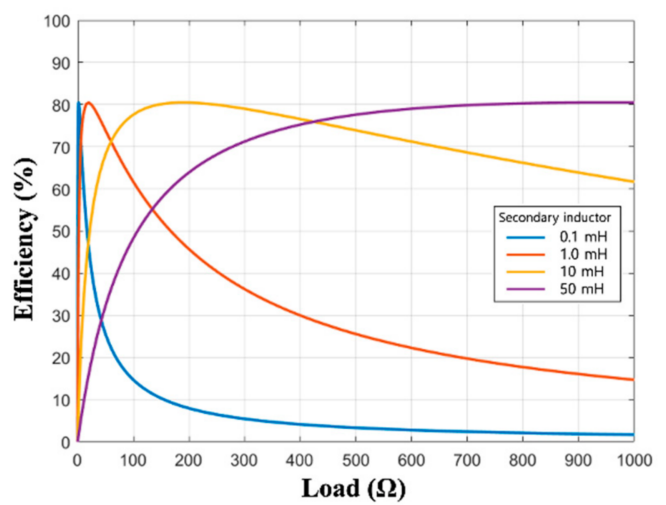

(b)

Figure 3. WPT characteristics respect to load resistance. (a) The required inductance of the secondary resonator for maximum efficiency. (b) Power transfer efficiency is displayed for different secondary inductance values.

It can be seen as an example that when the load resistance is $50 \Omega$, the optimal required inductance has to be more than 10 times the original value. Implementation of a coil with inductance in this range usually requires ferrite material and results in a bulky system. There are other matching techniques in existing literatures to maximize power transfer 
efficiency, but these techniques are based on radio frequency power amplifiers, in which the maximum deliverable power is typically below $10 \mathrm{~W}[5,7]$.

\section{Proposed WPT System Design}

In order to optimize the transfer efficiency under various loading condition, we propose a tunable capacitor network in the secondary resonator (Figure 4).

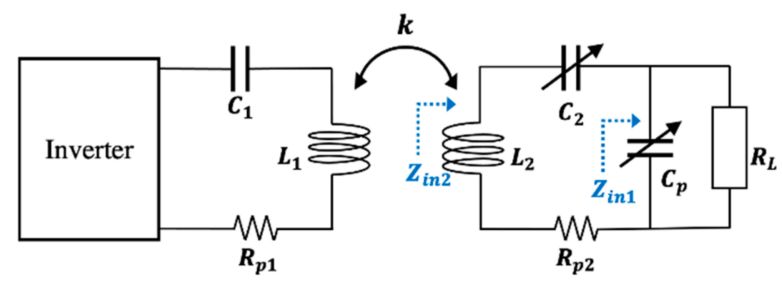

Figure 4. Schematic of the proposed WPT system.

Addition of a capacitive element parallel to $R_{L}$ can reduce the resistance of the impedance $Z_{\text {in } 1}$. From Figure 2, we know that the efficiency can be maximized if we can convert the impedance, $Z_{i n 1}$, to an optimum value. Assuming that $C_{2}$ is fixed, satisfying the resonant condition, $f_{0}=1 / 2 \pi \sqrt{L_{2} C_{2}}$, the following equations can be derived.

$$
\begin{gathered}
Z_{\text {in } 1}=R_{L} \| \frac{1}{j \omega C_{p}}=\frac{R_{L}}{1+\omega^{2} R_{L}^{2} C_{p}^{2}}-j \frac{\omega R_{L}^{2} C_{p}}{1+\omega^{2} R_{L}^{2} C_{p}^{2}} \\
\operatorname{Re}\left(Z_{i n 1}\right)=\frac{R_{L}}{1+\omega^{2} C_{p}^{2} R_{L}^{2}}=1.9 \Omega \\
\operatorname{Im}\left(Z_{i n 2}\right)=-\frac{\omega R_{L}^{2} C_{p}}{1+\omega^{2} R_{L}^{2} C_{p}^{2}}-\frac{1}{\omega C_{2}}+\omega L_{2}=0 \Omega .
\end{gathered}
$$

When the coil has inductance and quality factor of $100 \mu \mathrm{H}$ and 460 , respectively, the transfer efficiency is maximized when the resistance and reactance of $Z_{i n 1}$ becomes $1.9 \Omega$ and $0 \Omega$, respectively. Note that as the load resistance increases, the required $C_{p}$ decreases. Figure 5 illustrates the calculated power transfer efficiency respect to frequency. Without the use of any complicated compensation circuit or DC-DC converter system, the capacitive loading technique can significantly increase the power transfer efficiency.

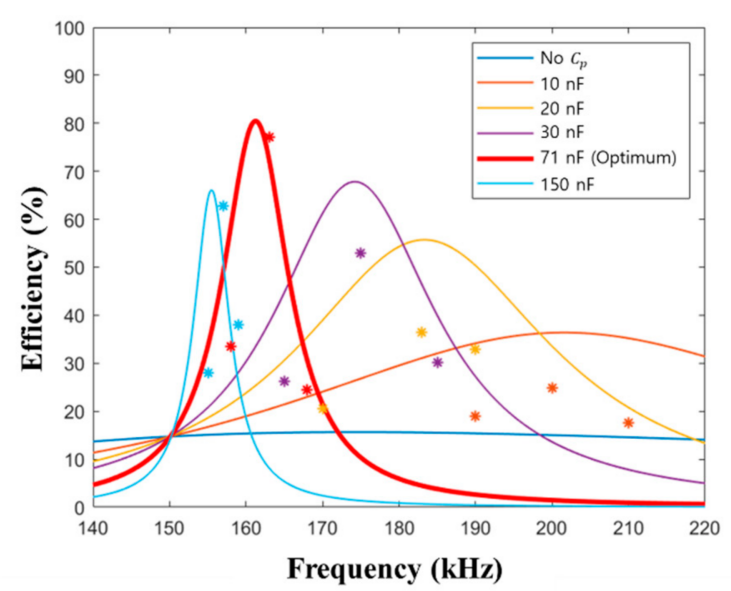

(a)

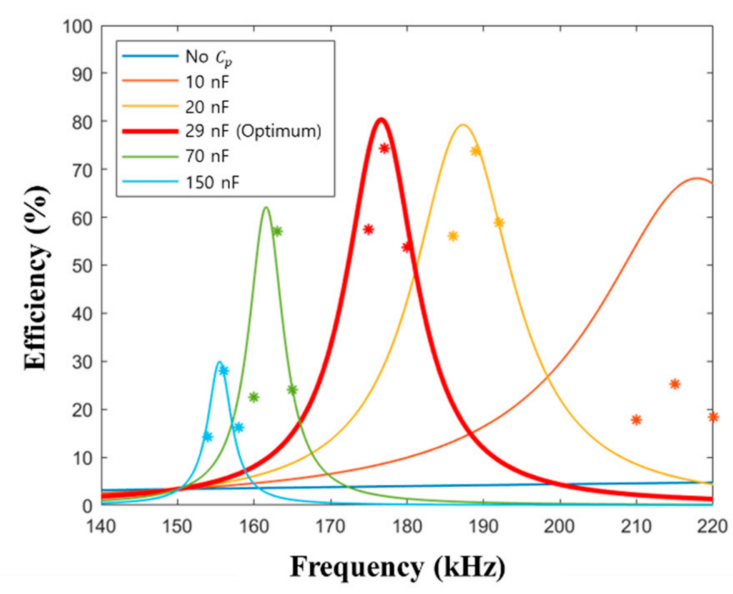

(b)

Figure 5. Power transfer efficiency as a function of frequency with different $C_{p}$ condition. (a) $R_{L}=100 \Omega,(\mathbf{b}) R_{L}=500 \Omega$. 
Even though the transfer efficiency can be maximized by employing $C_{p}$, the resonant frequency of the secondary coil network increases due to the additional parallel capacitance. The frequency shift can be calculated by the following equation.

$$
\omega^{2} L_{2} C_{p}-\frac{C_{p}}{C_{2}}-\frac{\omega^{2} R_{L}^{2} C_{p}^{2}}{1+\omega^{2} R_{L}^{2} C_{p}^{2}}=0
$$

Assuming that the third term can be approximated to 1, (15) can be reduced to (16).

$$
\omega^{2}=\frac{1}{L_{2}}\left(\frac{1}{C_{2}}+\frac{1}{C_{p}}\right)
$$

Since there is a noticeable frequency detuning at the secondary resonator, the output power available to $R_{L}$ is significantly decreased [21]. The amount of power delivered to the load when the maximum power transfer efficiency occurs at $161 \mathrm{kHz}$ is shown in Figure 6. Although the efficiency is maximized at $161 \mathrm{kHz}$, the available power to the load is very small. On the other hand, considerable power can be delivered when the system is operated at $150 \mathrm{kHz}$, but the power transfer efficiency is less than $15 \%$, suggesting that this design is not suitable for practical use.

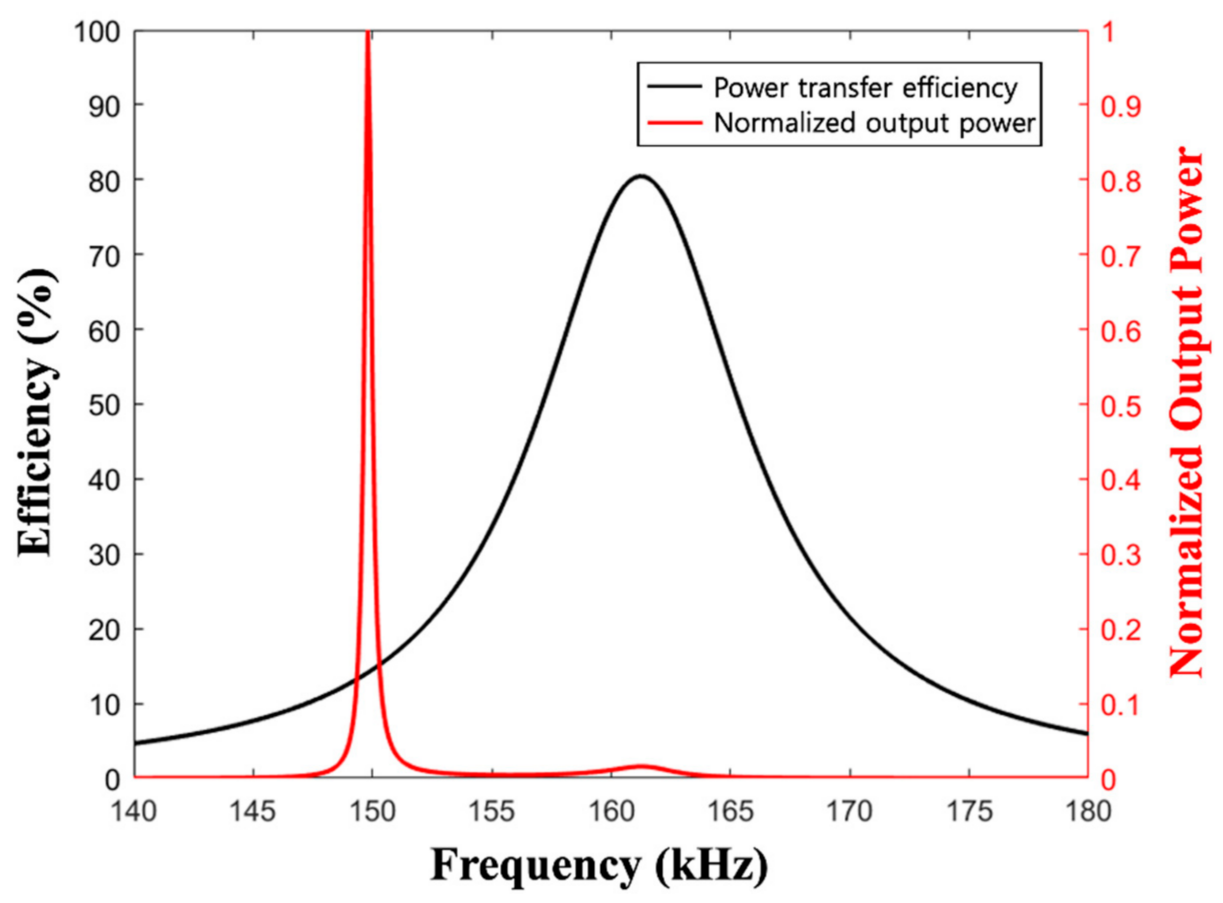

Figure 6. Calculated efficiency and normalized output power (w.r.t. its maximum value) as a function of frequency when $C_{p}$ is optimized to a $100 \Omega$ load.

In order to simultaneously coordinate the transfer efficiency and power delivery, the resonant frequency of the primary and secondary resonators should be designed at an equal frequency, $f_{0}=\omega_{0} / 2 \pi=150 \mathrm{kHz}$. The resonant frequency of the secondary resonator can be tuned back to $150 \mathrm{kHz}$ by manipulating the series capacitance $C_{2}$. Solving for the reactance of the secondary resonator, the required $C_{2}$ and $C_{p}$ can be obtained by (17)-(19). The detailed design process flow chart is shown in Figure 7. 


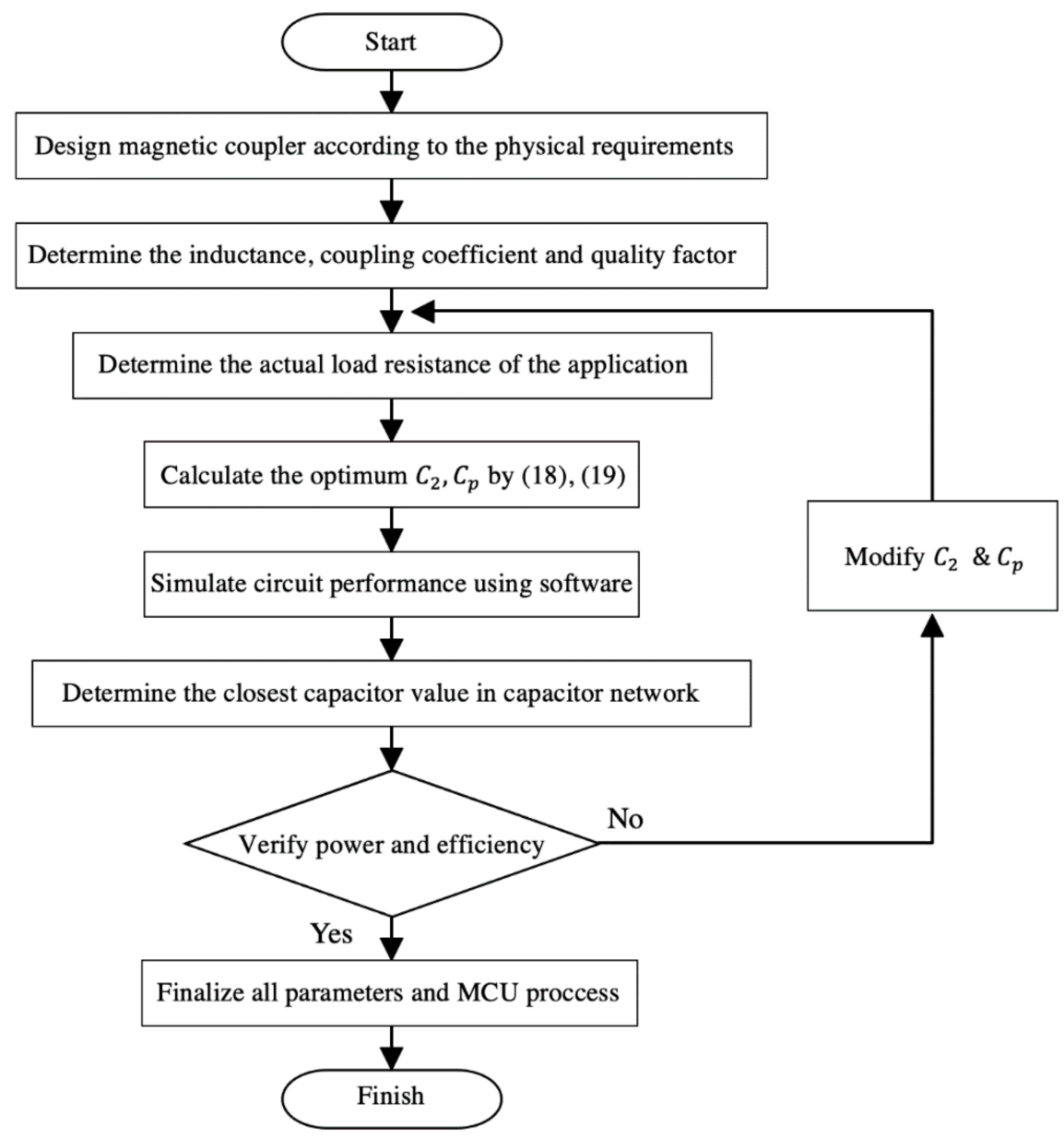

Figure 7. Design process of the proposed work.

$$
\begin{gathered}
\operatorname{Im}\left(Z_{i n 2}\right)=\omega_{0} L_{2}-\frac{\omega_{0} R_{L}^{2} C_{p}}{1+\omega_{0}^{2} R_{L}^{2} C_{p}^{2}}-\frac{1}{\omega_{0} C_{2}}=0 \\
C_{2}=\frac{1}{\omega_{0}^{2} L_{2}-\frac{\omega_{0}^{2} R_{L}^{2} C_{p}}{1+\omega_{0}^{2} R_{L}^{2} C_{p}^{2}}} \\
C_{p}=\frac{1}{\omega_{0} R_{L}} \sqrt{\frac{R_{L}}{1.9}-1}
\end{gathered}
$$

\section{Experimental Verification}

In this work, the coils were implemented using a 360-strand AWG-42 Litz-wire and both were constructed identically. The coil was made up of two stacks of 12-turn circular coils. The inner and outer diameters of the coil were $100 \mathrm{~mm}$ and $150 \mathrm{~mm}$, respectively. The coils were installed on acrylic sheets and the distance between the coils was arranged at $180 \mathrm{~mm}$ so that it could represent a loosely coupled scenario. The inductance, coupling coefficient and quality factor of the coils was measured at $150 \mathrm{kHz}$, with results identical to Section 3. An LCR (inductance, capacitance and resistance) meter (E4980AL, Keysight, Santa Rosa, CA, USA) was utilized to characterize all passive elements. A half-bridge inverter, digital signal processor (TM28335, Texas Instrument, Dallas, TX, USA) and a DC power supply (PWR041ML, Kikusui, Yokohama, Japan) were utilized to generate the $150 \mathrm{kHz}$ power source. Series and parallel connected power resistors were used to realize the various load resistance. The input power was obtained by measuring the power 
consumption of the inverter using a power analyzer (WT333E, Yokogawa, Tokyo, Japan) and the output power was determined by measuring the voltage present at the load. Power and efficiency measurements were taken by manually varying the load resistance and configuring the capacitance values to the optimum values.

The measured transfer efficiency of the proposed system by capacitance tuning is shown in Figure 8. The power transfer efficiency was determined by considering the DC input power supplied to the inverter and the output power delivered to the load. Even as the load resistance changed, the power transfer efficiency was maintained close to the theoretical maximum. Most of the values closely followed the calculated value from MATLAB at values near $150 \mathrm{kHz}$ but there existed some discrepancy at higher frequencies (Figure 5). The main reason for such discrepancy is due to the fact that the frequency dependency characteristics of passive elements (inductance, quality factor, coupling coefficients, etc.) and the switching loss associated with the power transistors in the inverter circuit were not taken into account in the calculation. The switching loss at the inverter is usually very small compared to the transferred power when dealing with more than $10 \mathrm{~W}$ but when the operating frequency is much larger than the resonant frequency of the primary resonator, the total output power becomes so small that the switching power loss becomes significant, further reducing the transfer efficiency. The power delivered to the load was verified up to $50 \mathrm{~W}$. It can be seen from Figure 9 that as long as the coupling coefficient and input voltage remain the same, regardless of the load resistance, the output power is kept at a constant level.

To construct an adaptive reconfigurable system, we used a network of single-pole single-throw relays (SRD-5VDC-SL-C, Ningbo Songle Relay, Yuyao, China) and a microcontroller platform (ATmega328P, Microchip, Chandler, AZ, USA), as shown in Figure 10. As an effort to reduce the voltage stress across $C_{2}$, the capacitor element was divided into two separate series capacitors. Although the 4-channel capacitance network was not able to implement the optimum capacitance with high accuracy, the discrepancy in the measured efficiency was less than $5 \%$. Table 1 shows a comparison of the calculated ideal capacitance and the actual measured capacitance used in this experiment. A photograph of the experimental demonstration is shown in Figure 11.

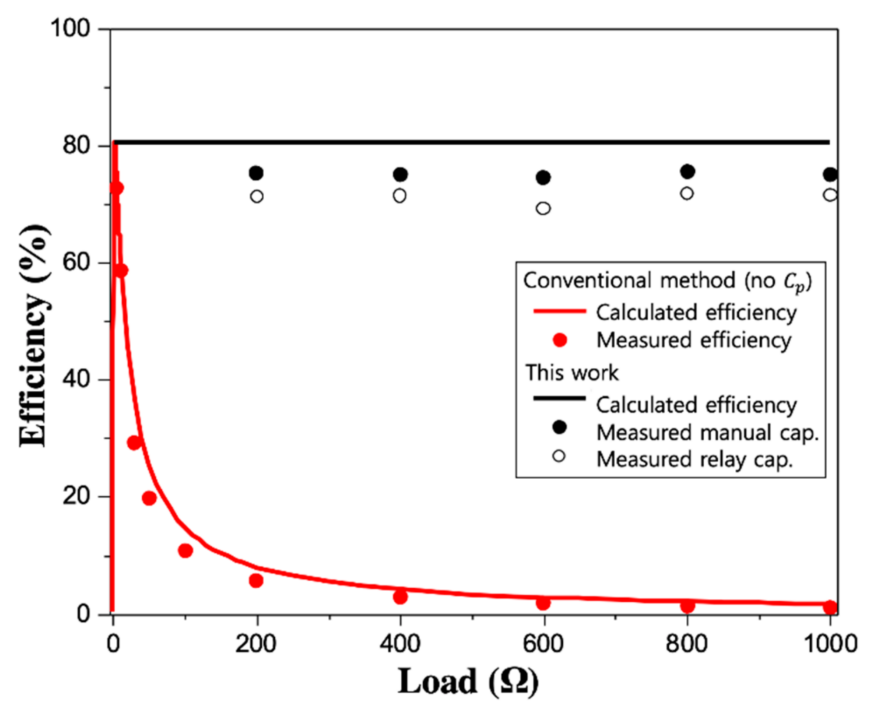

Figure 8. A plot of the transfer efficiency as a function of load. Solid line and the dot represent calculation and measurement, respectively. Note that the solid black dot is measured using manually optimized capacitors and the empty black dot is measured using 4-channel relay capacitor network. 


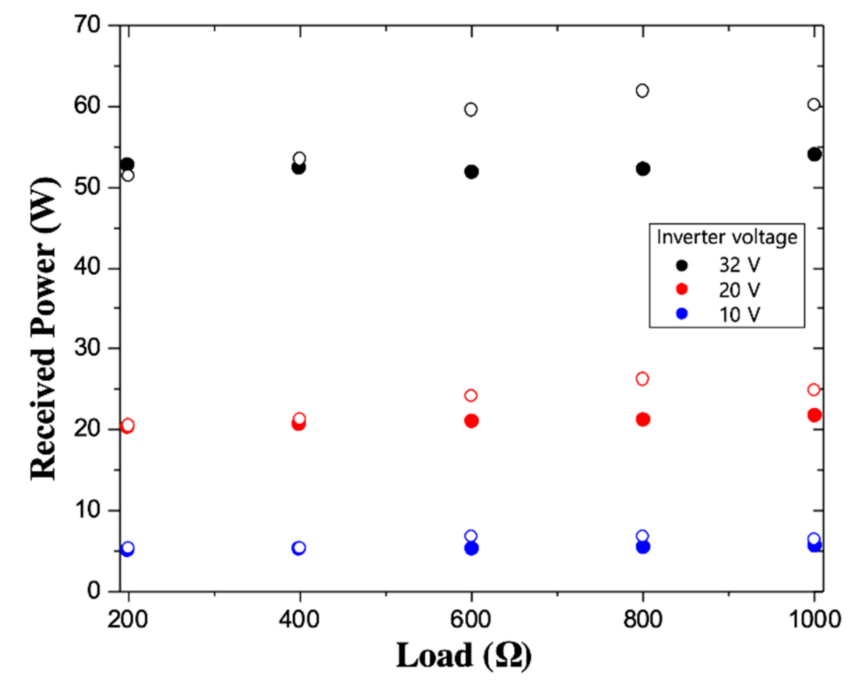

Figure 9. The output power at the load respect to load variation with different direct current (DC) voltage at inverter. The solid and empty dot denotes measurement using manually optimized capacitor and 4-channel relay capacitor network, respectively. Note that the amount of power delivered to the load does not depend on the load resistance.

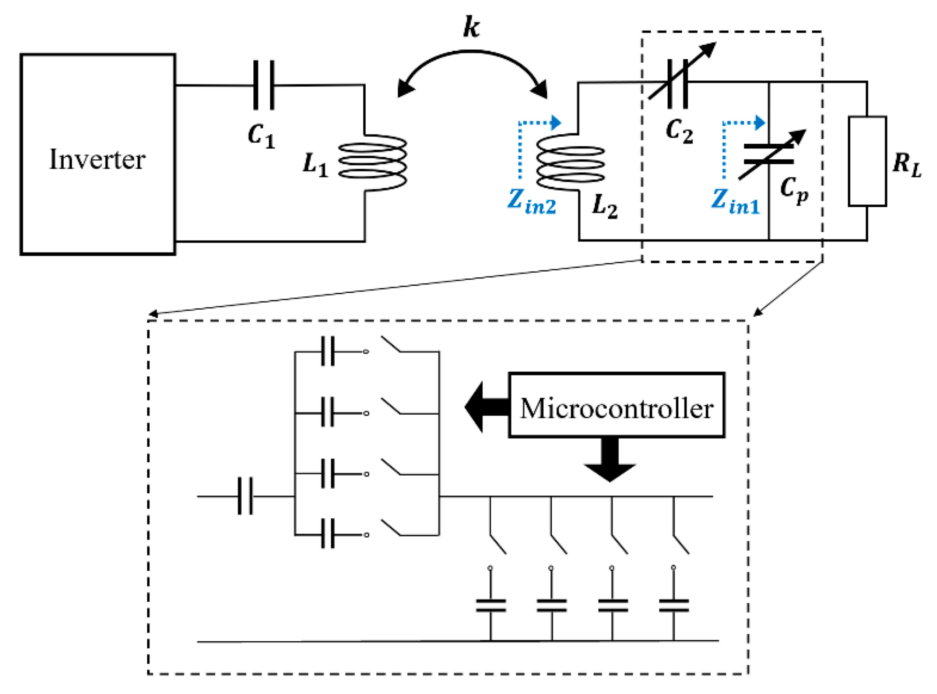

Figure 10. Proposed reconfigurable WPT system in this work.

Table 1. Capacitance of $C_{2}$ and $C_{p}$ used in this work.

\begin{tabular}{ccccccc}
\hline & \multicolumn{2}{c}{ Calculated Ideal Capacitance $(\mathrm{nF})$} & \multicolumn{2}{c}{$\begin{array}{c}\text { Manually Implemented } \\
\text { Capacitance }(\mathbf{n F})\end{array}$} & \multicolumn{2}{c}{$\begin{array}{c}\text { Capacitance Implemented } \\
\text { with Relay Network (nF) }\end{array}$} \\
\hline Load Resistance $(\boldsymbol{\Omega})$ & $\boldsymbol{C}_{\boldsymbol{p}}$ & $\boldsymbol{C}_{\mathbf{2}}$ & $\boldsymbol{C}_{\boldsymbol{p}}$ & $\boldsymbol{C}_{\mathbf{2}}$ & $\boldsymbol{C}_{\boldsymbol{p}}$ & $\boldsymbol{C}_{\mathbf{2}}$ \\
\hline 200 & 54.2 & 14.2 & 55.0 & 14.3 & 54.6 & 14.1 \\
400 & 38.4 & 15.9 & 38.6 & 16.0 & 37.1 & 16.1 \\
600 & 31.4 & 17.5 & 31.7 & 17.3 & 30.8 & 17.3 \\
800 & 27.2 & 19.2 & 27.3 & 24.9 & 20.2 \\
1000 & 24.3 & 20.9 & 24.3 & 20.9 & 24.9 & 20.2 \\
\hline
\end{tabular}




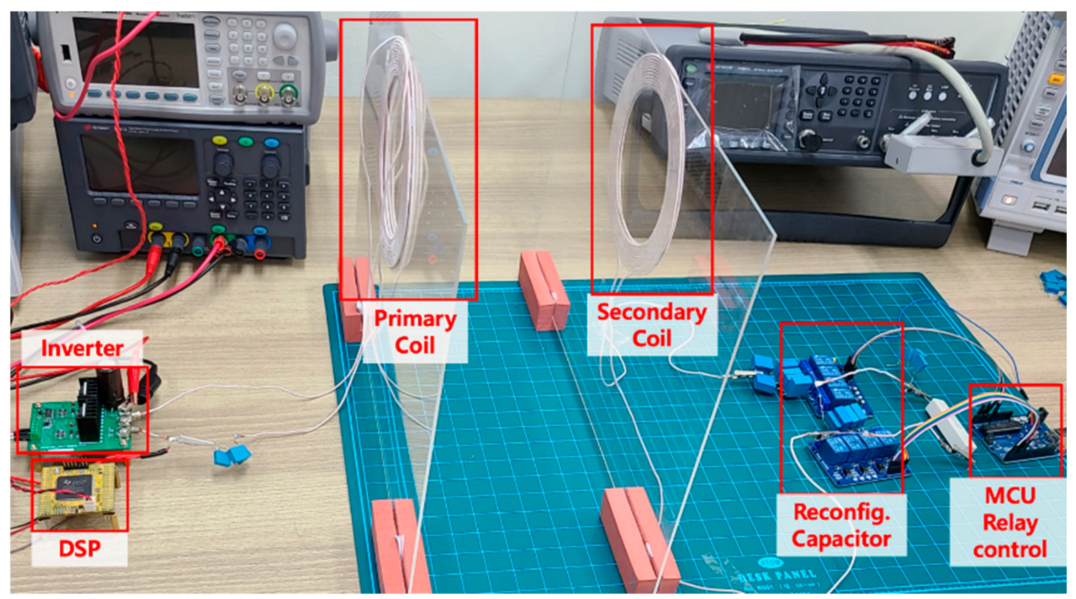

Figure 11. Photograph of the measurement setup.

\section{Conclusions}

In this work, a simple WPT solution for a loosely coupled, variable-load application is presented. A circuit model was first used to derive the optimal condition for a load variable environment. By adding an impedance transformation capacitance to the conventional series-series WPT system and following the design process, a power transfer efficiency close to the theoretical maximum was obtained. A detailed numerical derivation is provided to show the process to determine the optimal capacitive values. Calculation results show that maximum efficiency is achieved for a variable load condition. Experiments were carried out to verify the numerical analysis and the proposed approach. The proposed approach is simple, and the design is easily accessible for potential engineers in the wireless power transfer field. Further system miniaturization and precise value can be met by replacing the relays with bidirectional power switches. This proposed work suggests that applications with higher resistive load and distant power transfer range can effectively be wirelessly powered.

Author Contributions: J.-H.C. designed circuit models, performed computer aided calculation, conducted experimental measurement and wrote the initial draft; B.-H.L. designed the inverter, edited the manuscript and provided technical advice; Y.-J.K. proposed the initial design, edited the manuscript and led responsibility for the research activity. All authors have read and agreed to the published version of the manuscript.

Funding: This research was funded by the National Research Foundation of Korea (NRF-2019R1C1C1011235 and NRF-2020M3A9E4104385).

Institutional Review Board Statement: Not applicable.

Informed Consent Statement: Not applicable.

Data Availability Statement: Data sharing not applicable.

Conflicts of Interest: The authors declare no conflict of interest.

\section{References}

1. Hui, S.R. Past, present and future trends of non-radiative wireless power transfer. CPSS Trans. Power Electron. Appl. 2016, 1, 83-91. [CrossRef]

2. Xue, R.-F.; Cheng, K.-W.; Je, M. High-efficiency wireless power transfer for biomedical implants by optimal resonant load transformation. IEEE Trans. Circuits Syst. I Regul. Pap. 2012, 60, 867-874. [CrossRef]

3. Jayathurathnage, P.K.S.; Alphones, A.; Vilathgamuwa, D.M. Optimization of a wireless power transfer system with a repeater against load variations. IEEE Trans. Ind. Electron. 2017, 64, 7800-7809. [CrossRef]

4. Hui, S.Y.R.; Zhong, W.; Lee, C.K. A critical review of recent progress in mid-range wireless power transfer. IEEE Trans. Power Electron. 2014, 29, 4500-4511. [CrossRef] 
5. Kim, Y.-J.; Ha, D.; Chappell, W.J.; Irazoqui, P.P. Selective wireless power transfer for smart power distribution in a miniature-sized multiple-receiver system. IEEE Trans. Ind. Electron. 2016, 63, 1853-1862. [CrossRef]

6. Narayanamoorthi, R.; Vimala, A.J.; Chokkalingam, B.; Padmanaban, S.; Leonowicz, Z.M. Class E power amplifier design and optimization for the capacitive coupled wireless power transfer system in biomedical implants. Energies 2017, $10,1409$.

7. Koh, K.E.; Beh, T.C.; Imura, T.; Hori, Y. Impedance matching and power division using impedance inverter for wireless power transfer via magnetic resonant coupling. IEEE Trans. Ind. Appl. 2014, 50, 2061-2070. [CrossRef]

8. Kim, Y.J.; Bhamra, H.S.; Joseph, J.; Irazoqui, P.P. An Ultra-Low-Power RF Energy-Harvesting Transceiver for Multiple-Node Sensor Application. IEEE Trans. Circuits Syst. II Express Briefs 2015, 62, 1028-1032. [CrossRef]

9. Kim, Y.-J.; Maeng, J.; Irazoqui, P.P. Eyeglasses-powered, contact lens-like platform with high power transfer efficiency. Biomed. Microdevices 2015, 17, 1-9. [CrossRef]

10. Zhong, W.; Hui, S. Reconfigurable wireless power transfer systems with high energy efficiency over wide load range. IEEE Trans. Power Electron. 2017, 33, 6379-6390. [CrossRef]

11. Kim, J.; Jeong, J. Range-adaptive wireless power transfer using multiloop and tunable matching techniques. IEEE Trans. Ind. Electron. 2015, 62, 6233-6241. [CrossRef]

12. Darvish, P.; Mekhilef, S.; Illias, H.A.B. A novel S-S-LCLCC compensation for three-coil WPT to improve misalignment and energy efficiency stiffness of wireless charging system. IEEE Trans. Power Electron. 2020, 36, 1341-1355. [CrossRef]

13. Kim, D.-H.; Ahn, D. Self-tuning LCC inverter using PWM-controlled switched capacitor for inductive wireless power transfer. IEEE Trans. Ind. Electron. 2018, 66, 3983-3992. [CrossRef]

14. Liu, Y.; Feng, H. Maximum efficiency tracking control method for WPT system based on dynamic coupling coefficient identification and impedance matching network. IEEE Trans. Emerg. Sel. Top. Power Electron. 2019, 8, 3633-3643. [CrossRef]

15. Moon, S.; Kim, B.-C.; Cho, S.-Y.; Ahn, C.-H.; Moon, G.-W. Analysis and design of a wireless power transfer system with an intermediate coil for high efficiency. IEEE Trans. Ind. Electron. 2014, 61, 5861-5870. [CrossRef]

16. Brizi, D.; Stang, J.P.; Monorchio, A.; Lazzi, G. On the design of planar arrays of nonresonant coils for tunable wireless power transfer applications. IEEE Trans. Microw. Theory Tech. 2020, 68, 3814-3822. [CrossRef]

17. Dang, Z.; Cao, Y.; Qahouq, J.A.A. Reconfigurable magnetic resonance-coupled wireless power transfer system. IEEE Trans. Power Electron. 2015, 30, 6057-6069. [CrossRef]

18. Zhong, W.; Hui, S.Y.R. Maximum energy efficiency tracking for wireless power transfer systems. IEEE Trans. Power Electron. 2015, 30, 4025-4034. [CrossRef]

19. Fu, M.; Yin, H.; Liu, M.; Wang, Y.; Ma, C. A $6.78 \mathrm{MHz}$ multiple-receiver wireless power transfer system with constant output voltage and optimum efficiency. IEEE Trans. Power Electron. 2017, 33, 5330-5340. [CrossRef]

20. Low, Z.N.; Chinga, R.A.; Tseng, R.; Lin, J. Design and test of a high-power high-efficiency loosely coupled planar wireless power transfer system. IEEE Trans. Ind. Electron. 2008, 56, 1801-1812.

21. Mastri, F.; Costanzo, A.; Mongiardo, M. Coupling-independent wireless power transfer. IEEE Microw. Wirel. Compon. Lett. 2016, 26, 222-224. [CrossRef] 